\title{
5 Uncertainty in equilibrium
}

The existence of a problem of knowledge depends on the future being different from the past, while the possibility of the solution of the problem depends on the future being like the past.

Frank H. Knight (1921, p. 313)

In this chapter we develop the implications of real time for the important ideas of uncertainty and equilibrium. As we have already seen, the "genuine uncertainty" of real time is of a more basic and thoroughgoing nature than that usually treated in neoclassical models. The most important features of genuine uncertainty are the inherent unlistability of all possible outcomes resulting from a course of action, and the complete endogeneity of the uncertainty. The first feature, discussed in the last chapter, is the basis of novelty or true surprise. This is in sharp contrast to the mere arrangement (or weighting) of known possibilities characteristic of neoclassical uncertainty. The second feature, analyzed in the current chapter, is the origin of an ongoing market process that itself produces changes to which the system must adapt. The state of complete adaptation or equilibrium, by contrast, is preeminently a Newtonian concept. The absence of any tendency to change is incompatible with real time. Yet, as we shall see, some idea of equilibrium is important. Indeed, it would be difficult to imagine a viable economics without one. Thus, reconciliation between real time and equilibrium is necessary. A suitably reformulated equilibrium construct can be consistent with our realtime framework, and can also be the analytical source of the uncertainty and endogenous changes that pervade market processes. Real time, genuine uncertainty, and equilibrium all have important functions to perform in the analysis of an economic system. The detailed elaboration of this point is the task that we have set ourselves.

This chapter is divided into four sections. In the first, we examine both the endogeneity of uncertainty in real time and its incompatibility with standard notions of equilibrium. In the second section we analyze the anticipation of future events from the perspective of their typical and unique features. It will be shown that neoclassical economics deals with the typical and totally

DOI: $10.4324 / 9781315776736-10$ 
neglects the unique. The third section is a detailed examination of the equilibrium construct. The idea of exact equilibrium will be shown to be inadequate for understanding economic processes in real time. In its place, we advocate the idea of "pattern coordination." Finally, we explore the interrelation between equilibrium and optimality. Static suboptimality is there revealed to be an unhelpful concept. A useful welfare economics can only be based on a fully dynamic and nondeterministic perspective.

\section{Genuine uncertainty}

One of the most important features of genuine uncertainty is its endogenous and, consequently, ineradicable nature. In principle, activities directed toward anticipating the future or overcoming uncertainty in a world of real time cannot be completely successful. The famous Keynesian beauty contest (Keynes, 1964, p. 156) is an excellent illustration of this point. (We also make use of it in our discussion of the business cycle.) Since Keynes' illustration lacks an overall stable pattern of outcomes, the actual result is entirely time-dependent and, in this sense, completely unique. We use the term "genuine uncertainty" to refer not only to this aspect of prediction but, more generally, to the recognition that all attempts to characterize the future involve both unique and recurrent ("typical") features. Keynes' illustration emphasizes that aspect of genuine uncertainty that differentiates it from Newtonian or neoclassical uncertainty. This is the pure (Bergsonian) case of complete time dependency.

A hundred photographs are reproduced in a newspaper. Each contestant must choose the six prettiest or handsomest faces. The winner will be that contestant whose choices most closely approximate those of "average opinion." The goal of each contestant is therefore not to choose the six most attractive to him or her (opinion ${ }_{1}$ ), or even to guess what average opinion believes to be the most attractive (opinion ${ }_{2}$ ). Rather, the object must be to guess what average opinion believes that average opinion will choose (opinion ${ }_{3}$ ). Thus, the goal is to guess opinion ${ }_{3}$, which is, in turn, opinion 4 . As we shall see, there is in principle no limit to the height of the levels of guessing and counterguessing. There is no logically sufficient reason to stop at any given point; all such stopping is, to a large extent, arbitrary or derived from a convention. We have more to say on this last point in Chapter 10.

The two features of Keynes' example that we shall explore in detail are its endogeneity and its inconsistency with static equilibrium.

\section{Endogeneity}

Suppose there is an agency that collects and disseminates the guesses before they are actually entered in the contest (see the similar technique in Frydman, 1982). Now people know for sure what the average guesses of opinion 3 are at $t_{1}$. If every individual merely duplicates this opinion ${ }_{4}$, then no one will win. The prize will be so diluted (let us assume) that it is not enough to offset 
the opportunity costs of engaging in the contest. Therefore, in an effort to improve his chances, each individual will revise his guesses. Consider that the individual could act in one of two ways: (1) he could choose those pictures the agency reveals as the average choice and receive a negligible payoff, or (2) he could deviate from those in the hope that others will do the same and he will approximate the new average choices. In the knowledge that at least some, if not all, will act in accordance with (2), all will, in fact, do so. Now, of course, the point is to outguess or outpredict the crowd on the nature of these revisions. Therefore, there is still uncertainty, despite the existence of the information-disseminating agency. Further information has not eliminated (or even, in this example, reduced) uncertainty but has merely transformed it to a higher level of counterguessing. ${ }^{1}$ If the game is played round after round, people's opinions will continually change and there will be no natural end to the process.

There are two factors in Keynes' example responsible for its endogeneity. First, because we are dealing with a contest, it is obviously important to each contestant that he predict better than all the others. Thus, he has an incentive to gather such information as will give him an advantage or prevent others from gaining one. Second, because the individual is making predictions of predictions rather than of tastes, resource availability, and so forth, the relevant information will be what others are predicting. Therefore, knowledge gained over time by market participants will necessarily affect the objects of each agent's prediction. These considerations enable us to conclude that the very activity designed to cope with uncertainty (i.e., the acquisition of knowledge) is responsible for its continued existence. As soon as one level of uncertainty is eliminated, another level is necessarily created to replace it.

At any point in time, the uncertainty of this example can be seen in purely Newtonian terms. Every individual might be viewed as having a probability distribution over the possible guesses of average opinion. If we were to stop here, this would be no different than the uncertainty about tomorrow's weather or the outcome of some conventional game of chance. On the other hand, the important aspects of the example are only evident in the passage of time. From that perspective it becomes clear that we do not have the interaction of stable elements (like the isolated points of Newtonian time). The interaction itself ceaselessly changes those elements. As knowledge is allowed to increase, uncertainty is not eliminated but transformed.

\section{Inconsistency with equilibrium}

Purely time-dependent uncertainty is completely inconsistent with static equilibrium. In our example of this uncertainty there is no configuration of the "data" that is without a tendency to change. As long as time is allowed to pass, knowledge will grow and there will be endogenously produced change. ${ }^{2}$ Accordingly, it is not possible for the system to settle down to an equilibrium, whether of the exact or stochastic variety. Individuals always have a private 
incentive to overcome remaining uncertainty. As we have seen, however, the collective result of the actions undertaken on the basis of this incentive is to transform, rather than eliminate, uncertainty.

In a recent article Roman Frydman (1982) applies Keynes' beauty contest to a model that examines the possibility of convergence to rational expectations equilibrium. Not surprisingly, Frydman finds that under no plausible set of assumptions can we characterize a learning process that leads to an equilibrium. Such a process would have to involve forming expectations about the expectations of others (O'Driscoll, 1979, p. 162; see Rizzo, 1979, p. 11). Frydman's point, like ours, is that there is a form of uncertainty that cannot be eradicated by further knowledge. This is the time-dependent aspect of genuine uncertainty.

The critical contrast is therefore not, as some have suggested, between measurable and unmeasurable uncertainty (Knight, 1971), or even between subjective and objective interpretations of probability (Savage, 1972). It is instead between purely time-dependent and Newtonian forms of uncertainty. The former, as we have seen, is endogenously created and thus inconsistent with equilibrium, while the latter is essentially static and thus consistent with stochastic models of equilibrium. Neoclassical tools for handling uncertainty have been developed in the context of these models and consequently are adapted solely to the Newtonian perspective. To the extent that economists wish to construct models embodying time-dependent uncertainty, it is clear that new analytical tools will have to be developed. ${ }^{3}$

An important implication of time-dependent uncertainty is that exchanges will not take place at equilibrium prices. If the economic system endogenously produces disruptive forces, then we can no longer abstract from the problems of false trading. Instead of focusing our attention on the configuration of equilibrium exchanges or even the process of adjustment toward an equilibrium, the emphasis will shift to the pattern of disequilibrium exchanges. The "Austrian" business cycle theory is an example of systematic disequilibrium trading that results in a particular pattern of discoordinative behavior. This shall be examined in our chapter on money. Other attempts to study "disequilibrium patterns" have been reviewed by E. R. Weintraub (1979, pp. 141-54). The importance of these models lies in making the informational needs of agents quite specific. Since these needs cannot be fully satisfied by false prices, our attention is naturally shifted to the institutional framework and its role in promoting (or inhibiting) the coordination of plans. Nonprice conveyance of information is a necessary foundation for the process of monetary exchanges through time. We shall have more to say on this matter in the chapters on competition and money.

\section{Genuine uncertainty: typicality and uniqueness}

The purely time-dependent form of uncertainty discussed in the previous section must be an incomplete characterization of the way in which individuals 
anticipate the future. If the future were completely unpredictable in all respects, then planning and acting would be impossible. While complete stability and predictability are incompatible with time, their total absence is incompatible with action (Shackle, 1969, pp. 3-7). A stable framework within which it is possible to project consequences is a logical prerequisite for purposefulness. The solution to this paradox of uncertainty lies in the recognition of typical and unique aspects of future events. Genuine uncertainty is characterized by both relatively time-independent and time-dependent features in the flow of events. The crucial difficulty with neoclassical economics is its exclusive preoccupation with the former.

\section{Typification}

Typification is the activity that enables us to grapple with an unknown future. It is the process of "extracting what stability and regularity there is in the flow of reality" (Bergson, 1946, p. 111). Stable features are called types (Schutz and Luckmann, 1973, pp. 229-41). The stability of types is not to be identified solely with those aspects of events that actually have been repeated in case after case. Rather, stability refers to those aspects that are repeatable, in the sense that they are not affected by the mere passage of time. Consider, for example, the prediction that there will be a police patrol tonight. This amounts to a pattern or type prediction because we are quite able to conceive of a patrol as persisting in some unchanged overall pattern through time. The precise route they will take, what they will find, how they will react to a bank robbery, and so on constitute the (relatively) unique or time-dependent features of an event. Even these can be further broken down into their typical and unique aspects. The exact form that any prediction takes is thus obviously dependent on the practical interests and conceptual framework of the predictor.

Typical aspects of events can be anticipated in one of two ways: either with certainty, or probabilistically. From the certainty perspective, the predictor simply asserts that there will be a police patrol. In the probabilistic perspective, on the other hand, the predictor associates with a whole set of mutually exclusive typical events a series of weights. Thus, the probability of a police patrol may be 0.4 , that of a gang brawl 0.2 and that of a delivery of illegal narcotics 0.4 . The typicality of the future events is no different in the second than in the first case; what is different is the way in which they are anticipated. Probabilistic anticipation is the "loose," as contrasted to rigid, determinism discussed in Chapter 2. Nevertheless, even loose determinism is fundamentally static in at least two respects. First, there is a stationarity of the alternative events through time. This, after all, is the meaning of typicality. Open-endedness in the characterization of the alternatives is not possible. Second, techniques such as modification of probabilities based on Bayes' Theorem permit only deterministic changes. Given the occurrence of a certain subsequent typical event, there is only one way the probability weights can be altered. 


\section{The economics of time and ignorance}

The extraction of types or patterns is made possible by factors that affect both the state of the environment and individual interaction. In the first category, the possibility of typification rests on the stability of physical laws, that is, on the relative determinism of the world on the macro-(Newtonian) level. On this, of course, we can add nothing to the existing scientific literature. In the second category, there are the mutually reinforcing and stabilizing effects of rules of thumb and certain kinds of creative activity. Rules of thumb are adopted by agents when the computational demands of maximization are excessive (Hey, 1981, pp. 252-3), or when the recognition of time-dependent uncertainty makes the informational demands of maximization impossible (Loasby, 1976, p. 217). In the latter situation the probability distributions required by agents for maximization of expected utility are seen as incomplete descriptions of the relevant uncertainty. In either event, when agents follow rules of thumb their behavior is more predictable. Under a wide variety of situations the same rule will be applied.

Furthermore, creative entrepreneurship, spurred on by profit opportunities, strives to make coordination of plans possible. By shouldering uncertainty (Knight, 1971), or by attempting to foresee the future more accurately, entrepreneurs reduce the effective amount of uncertainty facing others (Kirzner, 1982). This does not mean that entrepreneurial activity does not create some uncertainty of its own. It simply means that, relative to a world of no entrepreneurship, the changes that exogenously impinge on a system are less disruptive. Because of entrepreneurship there is more stability and regularity in the flow of events.

\section{Uniqueness}

The unique features of events are the nonrepeatable aspects or, in other words, the specific time-dependent variants of a stable pattern. Nonrepeatability emerges from an event's temporal "place value" (Schutz and Luckmann, 1973, p. 240), that is, from its order in the flow of events. From the experience of any given event we derive certain interpretatively relevant conceptual structures that modify any subsequent experience (Schutz and Luckmann, 1973, p. 240). Thus, any attempt to anticipate the unique aspects of an event changes their place value at least because the anticipation itself must now affect the eventual experience. This is endogeneity of genuine uncertainty about which we have been talking. Consideration of a future event's unique features also emphasizes the basic open-endedness of anticipation in real time. We anticipate events as to their typical features but we cannot fill in the "details" beforehand.

In contrast, neoclassical economics evades the distinction between uniqueness and typicality. Those aspects of events are conflated when each possible situation or outcome is "condensed" into a single scalar quantity. From this perspective we delude ourselves into thinking that we have fully characterized the event and thus eliminated the open-endedness of the future. In reality, we 
have only characterized a single typical feature of the event in question and ignored everything else.

Since events can never be predicted precisely but only predicted as to types, there are three important circumstances under which the individual's interpretative-predictive scheme can change. First, when the typical event is anticipated with certainty, it still may not occur: a police patrol need not materialize. If it does not, the individual will obviously revise his view of what is stable in the environment. This is similar to what is generally meant by the refutation of a theory: when a predicted typical event does not occur, the theory undergoes change. Second, when typical events are anticipated probabilistically any outcome may induce revisions in the agent's framework. This depends on whether the actual outcome causes the agent to believe that the underlying probability distribution is different than he had originally thought.

Finally, even when the typical event does occur, or when the occurrence of a given typical event out of a set of possibilities does not itself cause the perceived underlying probability distribution to change, the agent may be led to revise his scheme. The unique aspects of events (or, more exactly, what appeared unique relative to the initial framework) provide further "data points" for its improvement. ${ }^{4}$ Since types are, by definition, the relatively stable elements in the flow of time, they will change only slowly. In other words, the interpretative framework and the types derived from it will change so gradually as to maintain their stability relative to the changes in the unique elements. Ultimately, however, a new framework necessarily arises out of the old. The bifurcation of our anticipatory vision into unique as well as typical features means that even correct prediction carries with it an endogenous source of eventual change. This source is the inevitable filling in of the "details" as events actually occur. As long as there are open-ended anticipations there will always, in the course of time, be at least gradual change in the frameworks from which they are derived.

\section{Equilibrium}

In the last two sections we discussed the implications of real time for the characterization of uncertainty. We saw that, in a world of time and genuine uncertainty, there is ceaseless endogenous change. In this and subsequent sections we shall direct our attention to the seemingly contradictory idea of equilibrium. Equilibrium has traditionally been centered on the absence of endogenous change. It has been conceived as the state of affairs produced after all endogenous forces have fully worked themselves out. From this perspective, time and equilibrium are surely incompatible (Shackle, 1972, pp. 253-4). It would be a mistake, however, to insist that equilibrium entails the absence of any and all tendencies to change. The uncertainty framework developed in this book can be incorporated into an equilibrium construct in such a way as to allow for unpredictable change. As we shall see, an appropriately revised idea of equilibrium need not be inconsistent with real time. 


\section{Equilibrium as exact coordination}

Austrians generally follow Hayek (1937a) in thinking of equilibrium in terms of the compatibility of individual plans. Thus, if $\mathrm{A}$ intends to buy $x$ units of a good at $\$ y$ per unit, then B (or some group of B's) must intend to sell $x$ at $\$ y$. This is a situation of ex ante coordination. If equilibrium is to prevail, however, the intentions of the various parties must be based on the same set of expectations regarding the external data. A may plan to buy umbrellas tomorrow on the expectation that it will rain, while B may plan to sell umbrellas on the expectation that it will not. Under these circumstances, the plans of the relevant parties cannot in principle be carried out. There is no single state of the world in which both A and B will actually implement their plans. Accordingly, the state of ex ante coordination is not enough for equilibrium; there must also be no logical impossibility standing in the way of the actual consummation of intentions.

Hayekian equilibrium can be partial or general, and can prevail over the various "runs" of Marshallian time. The degree to which equilibrium requires the homogeneity of expectations can be seen by contrasting the long run with Hicksian temporary equilibrium. In the former, all individuals must base their plans on the same expectations over the indefinite future. Thus, there must be complete expectational homogeneity. In temporary equilibrium, on the other hand, plans to buy and sell may be based on conflicting expectations with respect to the farther future. In fact, the existence of purely speculative markets requires the divergence of expectations (Lachmann, 1978, p. 5). Bulls plan to buy on the expectation that the price will rise, and bears plan to sell on the expectation that it will fall.

Nevertheless, the attainment of temporary coordination requires that the intentions of buyers and sellers be based on the same expectations with respect to the trading day. Suppose individuals make their plans at the beginning of the day and implement them by the end of the day. The ability of all parties to carry out their plans requires that, for example, expectations with respect to the time of sale be homogeneous. Expectational heterogeneity with respect to the more distant future will not frustrate this temporary coordination. Hayekian equilibrium therefore must entail homogeneous expectations with respect to the time period within which equilibrium prevails. Outside of that period, however, expectations can, and sometimes must, be divergent.

The homogeneity of expectations does not imply that they will turn out to be correct. Coordinative equilibrium is fundamentally a subjectivist construct and does not require consistency with the objective external data. If the expectations on which both parties base their intentions prove to be incorrect, then we say that the data have changed. Indeed "change" in a subjectivist framework can only be defined relative to the state of expectations (Hayek, 1973a, p. 40). Equilibrium is thus perfectly consistent with error. Error, once discovered, will however cause the equilibrium position to change. 
The consistency of Hayekian equilibrium with error provides the foundation for a wider appreciation of its subjectivist character. The importance of expectations means that equilibrium cannot be defined without reference to the interpretative-predictive schemes held by individuals. When these change so does the equilibrium. Furthermore, the objective possibilities, stressed by neoclassical economics as a codeterminant of equilibrium, are often not a binding constraint. In a world of divided knowledge, there is no single objective state of technique that governs the entire system. What the individual has learned is the constraint, and not all individuals will have learned the same things. Far from being an objective "pull" on the system, equilibrium makes sense only in the context of the presumed knowledge and expectations of economic agents. Equilibrium need not be a holdover from objectivist Ricardian economics. Instead, it can be seen as a particular relationship among myriad individual minds.

\section{Inadequacy of exact coordination}

Hayek's avowed intention in developing his concept of equilibrium as the consistency of individual plans was to marry time and equilibrium (Hayek, $1937 \mathrm{a}$, p. 37). Since plans are forward-looking, he reasoned that plan coordination must entail time. Unfortunately, he did not fully understand the distinction between the Newtonian and real-time constructs. Hayekian equilibrium incorporated only Newtonian time. Nevertheless, Hayek and the other Austrians did realize that equilibrium is not a directly operational construct and that the real world was never in equilibrium. They saw, however, at least two empirical uses for the concept of exact equilibrium. One was that it constitutes a research agenda; that is, it points to those factors worthy of further consideration. The other was a more sweeping claim: by reference to the construct, it was believed that we could predict the direction of realworld changes. In the following subsections we shall analyze these two views. Our examination of the purported uses of exact equilibrium will reveal its ultimate inadequacy.

Research agenda Mises developed what he called an argumentum a contrario (1966, p. 250). According to this, the equilibrium construct can be used as a foil against which to compare actual market situations. Thus, if conditions $\mathrm{a}, \mathrm{b}$ and $\mathrm{c}$ together imply a certain equilibrium, then the absence of that equilibrium would imply that at least one of these conditions does not hold. Economic analysis then focuses on the forces responsible for this situation. Consequently, from Mises' perspective, the exact equilibrium construct merely provides us with a clue as to why certain results do not obtain. Hence, it is capable of only negative prediction of states of affairs. It does not elucidate the actual processes by which those states may be achieved. It points us in the direction of those countervailing forces that are responsible for the actual outcome being different from the equilibrium outcome. Thus, exact equilibrium elucidates not the actual processes themselves but only the reason 
they do not produce a certain result. Hence it is fundamentally capable of only negative prediction.

Mises, however, claimed too much. This method does not permit us to offer a logically sufficient explanation for the "failure" of actual processes. For this to be the case, the conditions of equilibrium would have to be necessary conditions, whereas, in fact, they are merely sufficient conditions (Hausman, 1981, p. 152). The absence of sufficient conditions does not imply the absence of the result predicted by the equilibrium construct (e.g., Pareto optimality). Only the absence of all possible sets of sufficient conditions (the conjunction of which is a necessary condition) would imply this. Therefore, the foil method provides only part of the total explanation for the observed phenomena. Such partial explanations are also consistent with outcomes other than those observed, because sometimes the other sufficient conditions will be present and sometimes they will be absent. Hence an element of indeterminism is introduced into the explanatory process.

Direction of change In contrast to Mises, Hayek argued that equilibrium could be useful in making positive predictions. Indeed he contended that "the statement of the conditions under which individual plans will be compatible is ... implicitly a statement of what will happen if they are not compatible" (Hayek, 1941, p. 23; emphasis added). If all other prices are at their equilibrium levels and the price of apples is too high, relative to the price of oranges, to equate supply and demand, then the equilibrium construct tells us that the price of apples will fall. Hayek immediately recognized that, when there are many deviations from equilibrium, the "correct" change will depend on what is assumed to happen in the other markets (1941, p. 23, n. 1). In general, this will make predicting the direction of change an extremely complex matter.

The prediction of the direction of real-world changes is actually a form of pattern prediction. We are saying that a result of the type predicted by the construct will happen. If, as in our previous example, the price of apples were too high, a predicted fall is compatible with a whole host of actual changes. The only thing that unifies these changes is that they are all consistent with the type "fall-in-price." Therefore, for all practical or applied purposes we have departed from the exact conception of equilibrium. Equilibrium is now a direction rather than a point. This departure, however, has proceeded purely informally and is akin to merely "accidental" observational error.

As we have seen, both the research agenda and direction-of-change interpretations of the exact equilibrium construct take account of its imperfect applicability. But is this enough? Should we maintain an essentially timeless equilibrium and view indeterminism as merely a feature of the model's application? Or must we incorporate the indeterminism arising from real time and genuine uncertainty into the construct itself? If we are interested in using the idea of equilibrium to model or elucidate processes in real time, then consigning indeterminism merely to the realm of application is not sufficient. It is impermissible to model such a process as culminating in an exact equilibrium 
for two related reasons. First, recall the Popper-Schick proofs of the impossibility of agents predicting their own behavior (Chapter 2). If agents are uncertain about what they will do in the future, then they will never make plans that are exactly coordinated with those of others. Commitments will then be of a general variety rather than pinpointed to the precise future behavior of others (even assuming that the latter could be predicted).

Second, Oskar Morgenstern demonstrated that the assumption of perfect foresight, a defining characteristic of exact equilibrium, is inconsistent with any equilibrating process. He showed that, when one individual's plan is dependent on that of another, perfect foresight will produce "an endless chain of reciprocally conjectural reactions and counter-reactions" (Morgenstern, 1935, p. 174). This was illustrated in the famous Holmes-Moriarty story that is worth quoting in full:

Sherlock Holmes, pursued by his opponent, Moriarty, leaves London for Dover. The train stops at a station on the way, and he alights there rather than travelling on to Dover. He has seen Moriarty at the railway station, recognizes that he is very clever and expects that Moriarty will take a faster special train in order to catch him in Dover. Holmes' anticipation turns out to be correct. But what if Moriarty had been still more clever, had estimated Holmes' mental abilities and had foreseen his actions accordingly? Then, obviously, he would have travelled to the intermediate station. Holmes, again, would have had to calculate that and he himself would have decided to go on to Dover. Whereupon, Moriarty would again have "reacted" differently. Because of so much thinking they might not have been able to act at all or the intellectually weaker of the two would have surrendered in the Victoria Station, since the whole flight would have become unnecessary.

(Morgenstern, 1935, pp. 173-4)

The equilibrium in this illustration would be a stable set of plans that might result in Holmes escaping or in his being captured. That aspect of the solution is unimportant (to us); what is important would be the absence of any tendency for the plans of the individuals to change. In equilibrium their plans would be coordinated in the sense that each is optimal given what the other individual is in fact planning. The moral of the Holmes-Moriarty story, however, is that perfect knowledge of each other's plans makes attaining such an equilibrium impossible. Neither individual will be satisfied with any tentatively adopted plan and so coordination cannot be achieved. ${ }^{5}$ Despite allegedly perfect foresight - or, rather, precisely because of it - the genuine uncertainty of the Keynesian beauty contest has reasserted itself.

Therefore, imperfect foresight is a necessary, although not sufficient, condition for a process to result in an equilibrium. This equilibrium cannot, however, be a position of exact coordination. A process in which there must be errors cannot, except by chance, culminate in an errorless equilibrium. Hence, 
if we are to retain the notion of equilibrium, we must incorporate the reality of error into the construct itself.

\section{Pattern coordination}

The inadequacy of exact Hayekian equilibrium for the analysis of processes in real time means that we are faced with two alternatives: either (1) revise the equilibrium construct so as to incorporate time and uncertainty, or (2) abandon equilibrium altogether. Since Austrians have never believed that the state of plan coordination is a direct description of the real world, the issue is not one of "realism"; instead, it is the usefulness of the idea as a tool of analysis.

In the broadest sense, equilibrium is inextricably linked to the causal mode of reasoning. An equilibrium is merely a state of affairs in which the exogenous disturbing forces or changes have completely worked themselves out (Machlup, 1958, p. 48). As a pure construct, it is thus a mental laboratory in which various causes can be isolated from one another and their effects fully traced. Therefore, we believe that some concept of equilibrium is an indispensable ingredient in all economic explanations.

The only feasible alternative is to revise our notion of equilibrium by taking account of time and uncertainty. This process has been started in the work of those neoclassical economists who utilize the idea of a stochastic equilibrium. We propose, however, a different construct: pattern coordination. This makes use of both the original Hayekian "compatibility of plans" and the distinction between typical and unique aspects of future events. The plans of individuals are in a pattern equilibrium if they are coordinated with respect to their typical features, even if their unique aspects fail to mesh.

Consider, as an illustration, Professors A and B, who teach in the same department and who plan to discuss their forthcoming jointly authored book. Their plans are coordinated with respect to the typical features of their activities if, for example, each expects the other to be in his office on the day he actually plans to be there. Since neither has decided his position on the book's central concern beforehand, the contents of their discussions can be seen as the unique feature. What they will say depends on the "insights" that will arise only in the course of conversation. These insights are surely time-dependent. The plans of A and B are coordinated, therefore, in the sense that each will come into the office on the proper day and at the proper time, but they are not coordinated in the sense that each has planned what to say to the other. There is an open-endedness to their plans that allows for spontaneity or novelty. This is a pattern coordination.

There is also a looser form of pattern coordination, which we can call "stochastic pattern coordination." In this case, the typical features of activities are probabilistically coordinated. Thus, in the above illustration, both A and B may "envisage" a probability distribution over the days of the week of the other coming into the office. They each decide when to come based on this 
probability distribution. Sometimes the typical aspect of their activities will mesh in the exact sense and sometimes they will not. Overall, however, each individual is doing the best he can under the circumstances and so an equilibrium has been attained. The exact contents of the discussions, when they do occur, remain time-dependent. Therefore, this aspect of each individual's plans is not subject to stochastic characterization and must be truly openended.

In a manner characteristic of Newtonian time constructs, the conception of exact equilibrium conflates plans with activities. The looser idea, which we have called pattern coordination, involves the coordination of plans but not of actual activities. Plans made in a world of real time, as we have seen, must be open-ended. They embody the typical features of prospective actions and events, while the details are "filled in" as actions and events come to pass. Thus, coordination can exist with respect to plans or the typical features of planned activities, but not with respect to the actual activities themselves. The latter are a complex of typical and unique features and are not stable in real time.

A broadened or wider view of causality underlies the use of pattern coordination as a tool of process analysis. In the exact conception of equilibrium, tracing out the full effects of a change means showing how that change brings about a precise result. In the pattern view, on the other hand, we show the "full" effects of a disturbance when we follow it out to a certain class of results. In this sense, the causal analysis is less "full" or complete than in the exact case. This very incompleteness, however, constitutes a major advantage of pattern coordination. Real time and genuine uncertainty are not, by construction, eliminated from the analysis.

Equilibrium, in our new, less rigid sense, does not entail the complete absence of all tendencies to change. As we have seen, some features are stable while others continue to vary. The relatively stable types or patterns are, in the short run, affected only by exogenous shocks to the system. In other words, something more than the mere passage of time is needed to induce pattern reorganization. The unique aspects of events, however, are time-dependent and hence change from within the system. These kinds of changes are what constitute the endogenous market process. ${ }^{6}$ In that process market participants acquire non-theoretical "knowledge of the particular circumstances of time and place" (Hayek, 1945, p. 521) as the future comes into existence. Although such knowledge cannot be anticipated in either an exact or a probabilistic sense, the essence of entrepreneurship is to attempt to "see" these developments in advance. Because this aspect of the future is, by definition, time-dependent, no logically sufficient basis can be given for any particular prediction. Therefore, the entrepreneurial predictions will appear to be creative or intuitive rather than strict implications of a model. The goal of the economist, therefore, must be to render entrepreneurial prediction and its derivative behavior "intelligible." As we have seen in Chapter 2, this means that the conceptual scheme attributed to the relevant mind construct 
must show how the predicted behavior is more likely given the scheme than otherwise.

The analysis of an individual dealing with unique features of future events must involve a nondistributional measure of uncertainty. We cannot distribute unity over a fixed set of possibilities and expect to capture the essential indeterminism or open-endedness of the future. Shackle (1969, pp. 67-85, 117-69) has developed a framework ("potential surprise") designed to cope with this aspect of expectation. We shall not here try to restate his position or the criticisms that have been offered (Shackle, 1969, pp. 86-108, 170-9). It is sufficient, however, to note that neither subjectivists nor anyone else has fully developed a formalism to deal with this problem. This is one of the most critical tasks that remain to be completed in order fully to implement a subjectivist research program.

There are, to be sure, certain similarities between our concept of pattern coordination and neoclassical stochastic equilibrium. Nevertheless, there is a fundamental distinction. The crux lies in the recognition of uniqueness. In a stochastic pattern equilibrium the typical features are probabilistically coordinated as in the conventional stochastic equilibrium. However, the neoclassical analysis ignores time dependency. In so doing, it reduces all ideas of uncertainty and inexact equilibrium to pure Newtonian constructs.

The usefulness of a nondeterministic conception of equilibrium lies in our ability to use it to model adjustments in a manner that does not foreordain their outcomes. As we shall see in the next chapter, the competitive process is a discovery procedure and thus, in principle, its results cannot be predicted. On the other hand, it is important to be able to say something about the major features of market processes. The idea of pattern coordination allows us to have it both ways: we can discuss adjustments in general (pattern) terms while recognizing the essential creativeness of market activities.

\section{Equilibrium and optimality}

\section{Knowledge requirement for equilibrium}

Unlike the general competitive equilibrium of Arrow and Debreu, a Hayekian equilibrium, in either its exact or its pattern form, is not necessarily Paretooptimal. Participants in any given exchange are not assumed to possess all of the knowledge in the system or, especially, the knowledge of the observer. The knowledge requirement for plan coordination is simply that expectations be correct in the sense that nothing agents are "bound" to learn in the execution of their plans will falsify those expectations (Hayek, 1937a, p. 55). In effect, this means that equilibrium requires the absence of the type of endogenous learning that would upset initial (exact or pattern) plans. There ought to be nothing in the original configuration of data that will bring about their undoing. What individuals are bound to learn in any given situation depends, of course, on what the model-builder puts into his construction. This, in turn, 
depends on the empirical appropriateness of his assumptions about learning. Presumably, if an individual plans to erect a skyscraper he will inevitably learn whether the ground underneath will support such a heavy structure. Moreover, we may require him to learn what other individuals will reveal or do in the course of executing his plans. Market activity, for example, may cause the price of certain types of labor and materials to rise before the skyscraper is completed. This could result in the project being left unfinished. Only if such information is correctly anticipated at the beginning of the period can there be plan coordination.

\section{Irrelevance of suboptimality in static equilibrium models}

A number of neoclassical economists have recognized that meaningful welfare economics cannot be based on Pareto optimality as the standard of evaluation. Suboptimality relative to knowledge possessed by an omniscient observer is completely irrelevant. In the absence of a feasible alternative institution that could remedy the problem, we have merely demonstrated that the world is imperfect (Demsetz, 1969). This, of course, will always be the case. Suboptimality relative to information possessed by some "isolated" individual in the system is also irrelevant in the absence of a tendency to dissemination. Dissemination may be impossible owing to the costs of acquiring information. If it is argued that optimality-producing information can, in some way, be provided to the system, there is an obvious puzzle. It is inexplicable, in a static context, that such information has not already been provided or is not already being provided at the optimal rate. Static models effectively abolish time and succession. All that eventually "will" happen happens now. Therefore, what started out as a suboptimal state of affairs is now revealed to have been an optimum all along. Standard equilibria are optimal with respect to the knowledge that the relevant agents do have. In other words, people make the best use of what they know (the "efficient markets" hypothesis). But this is trivial from a normative perspective: optimality here means nothing more or less than equilibrium. Pursuit of this line of reasoning would lead to the crude Panglossian tautology that has plagued the work of some economists (e.g., Stigler, 1982).

Only something exogenous to the model can account for the system's suboptimality. This is equivalent to saying that static maximizing models cannot explain (rationalize) suboptimality; they can merely postulate it. Either an equilibrium is suboptimal in an irrelevant and unexplained sense, or it is optimal in an explained but trivial sense. Static welfare economics thus self-destructs. As long as there are logically sufficient reasons for a given imperfect state of knowledge, we obviously cannot say that there could be less ignorance. The preexisting conditions (e.g., costs and benefits of search) rigorously imply that state of knowledge. Consequently, we must loosen the link between causes and results in order to say that things could be better or that more knowledge could prevail. As we have seen, a conceptual framework 


\section{The economics of time and ignorance}

that takes time seriously will introduce such indeterminism in the structure of models.

Thus, from the dynamic perspective developed in this book, there can be some role for welfare economics. Since knowledge would then be changing, we obviously could not evaluate a system by the efficiency of its allocations relative to some fixed body of knowledge. Instead, the only possible standard would be the degree to which various processes and systems generate knowledge or make discoveries. The competitive market, viewed as an engine of discovery, is the topic of the next chapter. In that chapter, we deal with welfare analysis in a dynamic theory.

\section{Notes}

1 This is not to deny that the uncertainty could be overcome for an individual if the agency were to reveal the guesses only to that individual. If no one else knew this, then there would be no incentive for anyone except the lucky person to change his guesses. This, however, would mean that we were back in a Newtonian world.

2 For the purposes of the model, time will stop if the agency calls an end to its activities and people are required to make their final guesses.

3 G. L. S. Shackle has made an important start in this direction. See especially Shackle (1969).

4 Although the framework may have predicted "well" in the past, it could have done better. In addition, circumstances may have changed and so the framework must also change in order to do equally well in the future.

5 Another example of the phenomenon was provided by G. B. Richardson (1960). Assume, initially, that in one industry there is an "initially" expected rate of return higher than the normal rate. Assume further that all market participants have perfect foresight of this and that all the other conventional assumptions of the competitive model have been satisfied. If each potential entrant must take into account the behavior of others because they can produce competitive supply, there will be either an indefinite expansion of output or no expansion at all. If everyone foresees a supernormal return, then an indefinitely large number of firms will enter at the same time and the actual rate of return will be indefinitely lower than the "initially" expected return. But if people are very clever they will foresee this and not enter. If, however, no one enters, then the rate will again fail to be bid down to the equilibrium level. In neither case, then, is the assumption of perfect foresight compatible with a tendency toward equilibrium. This is not a mere cobweb-like situation but rather a problem of logical circularity. Each potential entrant must make his decisions on the basis of what others decide. Perfect knowledge of what they intend to do, however, paralyzes the ability of each to make any decisions at all. Also cf. Frydman et al. (1982).

6 There is an "exogenous market process" only in the sense of a market reaction to exogenous shocks. Although the process may be initiated by something outside the system, the adjustment process itself is endogenous. 